\title{
Linfangioleiomiomatosis esporádica. Presentación de un caso con enfermedad pulmonar y linfangioleiomiomas retroperitoneales
}

\author{
Sporadic lymphangioleiomyomatosis. Presentation \\ of a case of pulmonary disease with retroperitoneal \\ lymphangioleiomyomas
}

Claudia Patricia Zuluaga Gómez, MD. ${ }^{(1)}$; Astrid Arroyave, MD. ${ }^{(2)}$; Diana Carolina Moreno O., MD. ${ }^{(3)}$; JuAn Ricardo LUtZ, MD.(4); JoRge Alberto CaRRILlo BayonA, MD.(5)

\section{Resumen}

La linfangioleiomiomatosis (LAM) es una enfermedad multisistémica asociada a mutaciones del gen TSC 2, que se presenta en forma esporádica (LMA-E) o asociada al complejo de esclerosis tuberosa (LAM-CET). La LAM cursa con enfermedad pulmonar relacionada con quistes en el parénquima. Sin embargo, los pacientes con LAM-E pueden evidenciar alteraciones mediastinales, retroperitoneales y renales, con adenomegalias, linfangioleiomiomas y angiomiolipomas.

Presentamos el caso de una paciente con LAM-E y enfermedad pulmonar quística asociada a linfangioleiomiomas retroperitoneales y pélvicos.

Palabras clave: linfangioleiomiomatosis, linfangioleiomiomas, angiomiolipomas, quistes.

\begin{abstract}
Lymphangioleiomyomatosis (LAM) is a multisystemic disease associated with mutations of the TSC 2 gene. It has two presentations: sporadic (S-LAM) and associated with the tuberous sclerosis complex (TSC-LAM). LAM runs together with pulmonary parenchymal cystic disease. However, patients with S-LAM can develop mediastinal, retroperitoneal and renal alterations, with adenomegaly, lymphangioleiomyomas, and angiomyolipomas.
\end{abstract}

We present the case of a female patient with S-LAM and cystic lung disease associated with retroperitoneal and pelvic lymphangioleiomyomas.

Keywords: lymphangioleiomyomatosis, lymphangioleiomyomas, angiomyolipomas, cysts.

\section{Caso}

Paciente de sexo femenino, de 43 años, con dolor torácico de inicio súbito y disnea. Antecedente de episodio similar 10 años antes con diagnóstico de neumotórax, tratado con toracostomía cerrada. Asintomática entre los episodios. La radiografía de tórax inicial evidenció neumotórax izquierdo y opacidades reticulares finas bilaterales, simétricas (figura 1). La torocastomía cerrada permitió una adecuada expansión pulmonar. La tomografía computarizada de alta resolución (TCAR) demostró lesiones de aspecto quístico en el parénquima pulmonar asociadas a cámara de neumotórax izquierdo (figura 2). En la tomografía computada (TC) de abdomen y pelvis se evidenciaron
(1)Médica Radióloga. Hospital Universitario Mayor Mederi. Universidad del Rosario.

${ }^{(2)}$ Fellow de Imagen Corporal. Universidad de Antioquia.

${ }^{(3)}$ Residente de Medicina Interna. Universidad del Rosario.

${ }^{(4)}$ Médico Neumólogo. Hospital Universitario Mayor Mederi. Universidad del Rosario.

${ }^{(5)}$ Médico Radiólogo. Hospital Universitario Mayor Méderi. Universidad del Rosario.

Correspondencia: Claudia Patricia Zuluaga Gómez, correo electrónico: patozulua@ gmail.com

Recibido: 16/04/15. Aceptado: 26/04/15. 


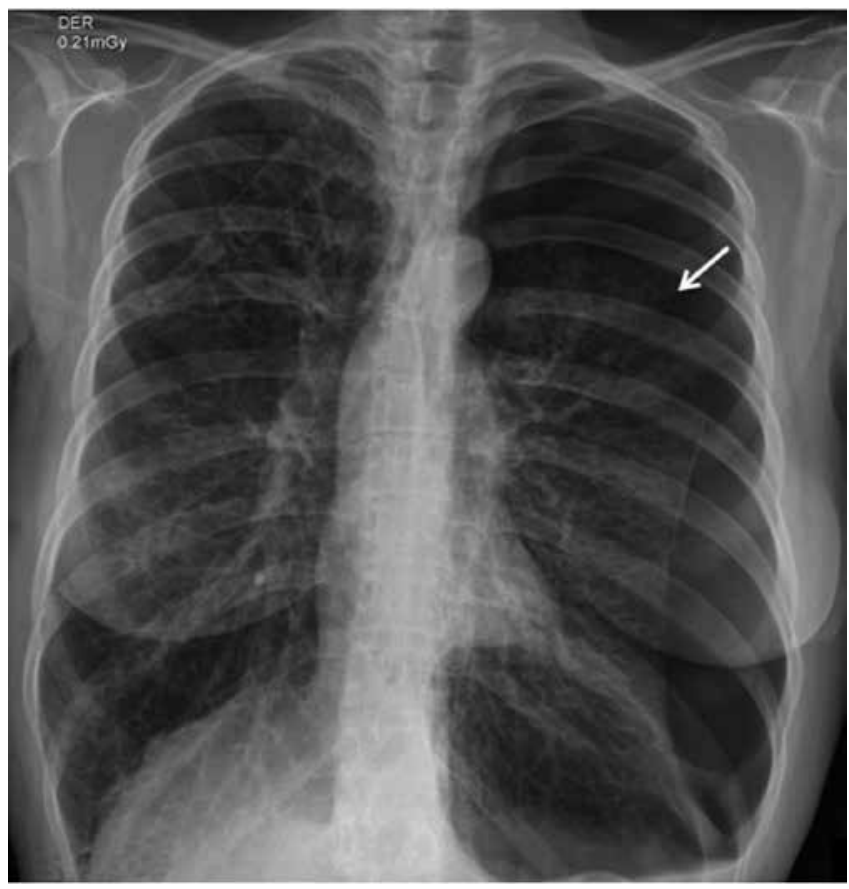

Figura 1. Radiografía de tórax. Opacidades reticulares finas bilaterales. Neumotórax izquierdo (flecha blanca). masas quísticas retroperitoneales y pélvicas interpretadas como linfangioleiomiomas (figura 3). Las pruebas de función pulmonar demostraron alteración severa en la DLCO (capacidad de difusión de monóxido de carbono) y un patrón mixto severo con mínima respuesta al broncodilatador. La resonancia magnética (RM) cerebral se consideró dentro de límites normales. Teniendo en cuenta el sexo y la edad de la paciente, la enfermedad actual, el antecedente de neumotórax y las alteraciones en los estudios de imágenes se consideró el diagnóstico de linfangioleiomiomatosis esporádica.

\section{Discusión}

La linfangioleiomiomatosis (LAM) es una enfermedad multisistémica poco frecuente, que afecta predominantemente a mujeres en edad fértil, con dos formas de presentación: a) esporádica (LAM-E) y b) asociada con el complejo de esclerosis tuberosa (LAM-CET). La LAM se caracteriza por la presencia de enfermedad pulmonar quística, angiomiolipomas extrapulmonares, tumores linfáticos y quilotórax.

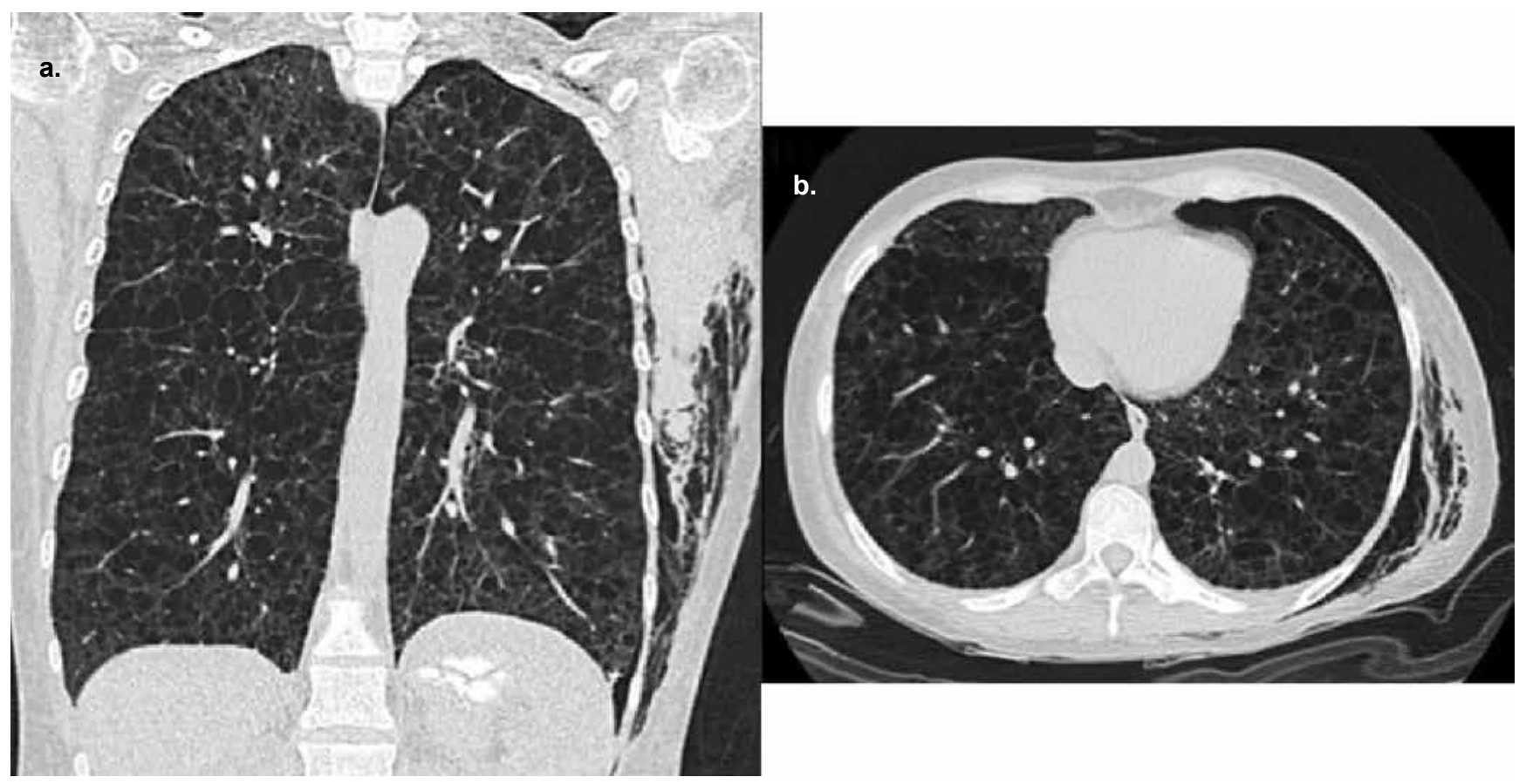

Figura 2. Tomografía computarizada de alta resolución de tórax. A. Reconstrucción coronal. B. Corte axial. Lesiones. 


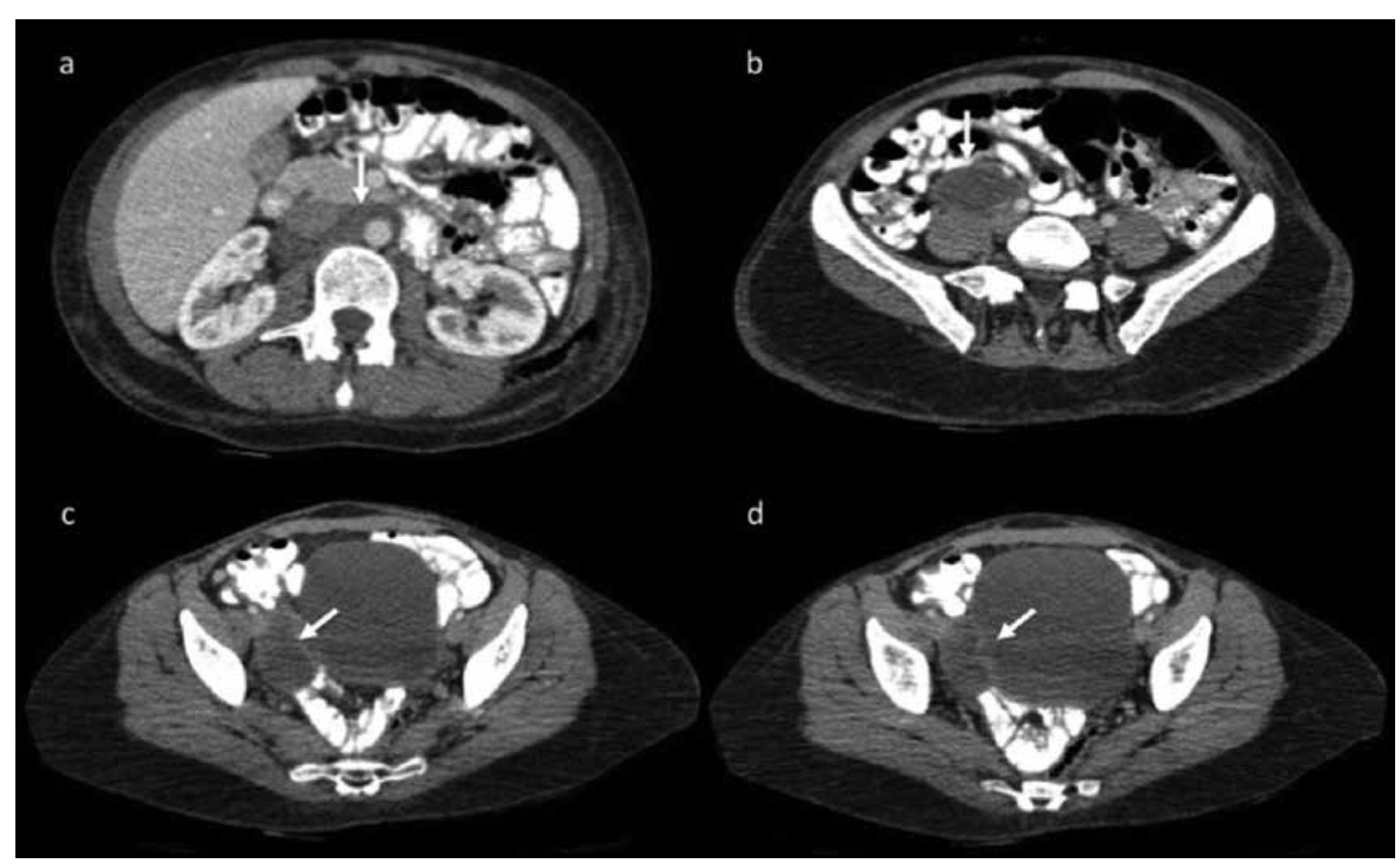

Figura 3. Tomografía computarizada de abdomen contrastada. Masas con densidad quística (flechas) retroperitoneales y pélvicas, de morfología redonda y contornos lisos compatibles con linfangioleiomiomas.

El complejo de esclerosis tuberosa (CET) es un síndrome neurocutáneo de transmisión autosómica dominante, causado por mutaciones en los genes TSC1 y TSC2 y caracterizado por retardo mental, autismo, convulsiones y lesiones hamartomatosas en cerebro, corazón, piel, riñones, ojos, pulmones e hígado (angiofibromas faciales, fibromas periungueales, rabdomiomas cardiacos y astrocitomas de células gigantes, entre otros) (1).

La asociación de esclerosis tuberosa y linfangioleiomiomatosis (LAM) es conocida en la literatura. Publicaciones recientes han evidenciado quistes pulmonares hasta en el $80 \%$ de mujeres con CET. En pacientes de sexo masculino la asociación LAMCET es de menor relevancia (13\% de los hombres con CET cursan con LAM) (2).

La LAM-E es causada por mutaciones somáticas en el gen TSC 2 con una prevalencia de 1 en 400.000 mujeres (1). Estos pacientes pueden tener manifestaciones extrapulmonares descritas en el CET que incluyen: angiomiolipomas, adenomegalias y linfangiomas abdominales, pero no presentan manifestaciones cutáneas, oculares o en el sistema nervioso central (3).

\section{Fisiopatología}

La LAM se caracteriza por la proliferación aberrante de células LAM (células inmaduras de tipo mesenquimal con características de células musculares lisas y melanocitos). Las células LAM presentan marcadores de diferenciación muscular como alfa-actina de músculo liso, miogenina y MyoD1. La característica principal de las células LAM es su positividad para $\mathrm{HMB} 45$, un anticuerpo monoclonal contra la proteína gp100 de los premelanosomas (3).

Se considera que existe un mecanismo fisiopatológico común entre el CET y la LAM. En ambas entidades se describen mutaciones en los genes TSC1 (Tuberous Sclerosis Complex 1), ubicado en el cromosoma 9q34 y TSC2 (Tuberous Sclerosis Complex 2) localizado en el cromosoma 16p13. Estos genes codifican para las proteínas hamartina y tuberina, encargadas de reducir el nivel de Rheb-GTP a través de la activación de una GTP-asa, con inhibición en 
el mTOR (mammalian target of rapamycin), un mediador de la proliferación y del crecimiento celular a través de la síntesis proteica (3-5).

Las células LAM proliferan e infiltran el parénquima pulmonar, los bronquios y los vasos sanguíneos y linfáticos (intra y extrapulmonares). La obstrucción de los bronquiolos terminales por células de músculo liso, genera atrapamiento aéreo con dilatación progresiva del espacio aéreo distal y formación de quistes pulmonares, que condicionan el deterioro progresivo de la función pulmonar $(6,7)$. También se describe hiperplasia multifocal micronodular de los neumocitos tipo 2, de mayor frecuencia en LAM-CET (12\%) que en LAM-E (1\%) (8-10). La infiltración de las paredes vasculares con obstrucción de los vasos sanguíneos puede condicionar edema y hemorragia pulmonar. El aumento de tamaño del conducto torácico se relaciona con depósito de células LAM en la pared del conducto, con estrechez y obstrucción secundaria, o ambas y dilatación de los linfáticos proximales (9). La obstrucción de los linfáticos (intra y extrapulmonares) es responsable de la presencia de adenomegalias, ascitis, quilotórax, quilopericardio y linfangioleimiomas (3).

\section{Clínica}

La LAM afecta a mujeres en edad reproductiva con una edad media de 34 años. Sin embargo, hay reportes de la enfermedad en mujeres postmenopaúsicas, en terapia de reemplazo hormonal y en hombres con el CET (1).

Las manifestaciones de la enfermedad pulmonar caracterizan el cuadro clínico de los pacientes con LAM e incluyen: disnea de progresión lenta, neumotórax a repetición (50-60\% de los pacientes) y/o tos improductiva. Con menor frecuencia pueden presentar hemoptisis y quilotórax (3).

Entre las manifestaciones extrapulmonares secundarias a la alteración de linfáticos retroperitoneales, mediastinales y pélvicos se encuentran: adenomegalias, linfangioleiomiomas (masas quísticas de crecimiento lento), angiomiolipomas (predominantemente renales) y ascitis quilosa (3).
En la LAM esporádica predomina la alteración de los linfáticos (dilatación del conducto torácico, derrame pleural quiloso, ascitis y linfangioleiomiomas) y en la LAM asociada a CET son característicos los nódulos pulmonares no calcificados y los angiomiolipomas (renales y hepáticos) (1).

\section{Diagnóstico}

El diagnóstico se basa en una presentación clínica típica (mujeres en edad fértil con disnea, neumotórax a repetición o quilotórax), hallazgos característicos en la TCAR e histología compatible (3). No obstante, en los criterios propuestos por la Sociedad Europea Respiratoria (ERS) del año 2010 (tabla 1) no se requiere biopsia pulmonar en los pacientes con manifestaciones características de LAM en la TCAR de tórax y uno de los siguientes criterios: angiomiolipomas, derrame quiloso, linfagioleiomiomas o esclerosis tuberosa (probable o definitiva). Una TCAR característica de LAM en ausencia de hallazgos extrapulmonares no se considera diagnóstica (11).

Un valor elevado de factor de crecimiento vascular endotelial D (VEGF-D) sérico tiene buena sensibilidad y especificidad para el diagnóstico de LAM. El punto de corte de 0,8 ng/ml tiene una sensibilidad del $73 \%$ y una especificidad del 100\% (12). Se estima que el $90 \%$ de las LAM probables por criterios ERS serían definitivas si se adicionara VEGF-D a los criterios diagnósticos (12). Estudios recientes han evaluado la utilidad del VEGF-D como factor pronóstico y para seguimiento de pacientes con LAM en tratamiento. Se describe una buena correlación entre los valores de VEGF-D, la severidad de la enfermedad y la respuesta al manejo con sirolimus (13).

En las pruebas de función pulmonar, la mitad de los pacientes con LAM presentan un patrón obstructivo, de los cuales un $25 \%$ tiene prueba de broncodilatador positiva y descenso en la DLCO. Los demás pacientes evidencian patrones normales o mixtos (3).

Los especímenes obtenidos por biopsia pulmonar o ganglionar, se caracterizan por focos de células 
Tabla 1. Criterios diagnósticos de LAM. Guías de la ERS 2010.

\begin{tabular}{|lll|}
\hline \multicolumn{1}{|c|}{ LAM defiitiva } & \multicolumn{1}{c|}{ LAM probable } & \multicolumn{1}{c|}{ LAM posible } \\
$\begin{array}{l}\text { 1. Hallazgos compatibles en TC de } \\
\text { tórax y biopsia }\end{array}$ & $\begin{array}{l}\text { 1. Caracteristicas en TC de tórax e } \\
\text { historia clinica compatible }\end{array}$ & $\begin{array}{l}\text { Características o hallazgos } \\
\text { compatibles en TC de tórax }\end{array}$ \\
$\begin{array}{lll}\text { 2. Hallazgos compatibles en TC de } \\
\text { tórax y uno de los siguientes: }\end{array}$ & $\begin{array}{l}\text { 2. Hallazgos compatibles en TC de } \\
\text { tórax y alguna de las siguientes: }\end{array}$ \\
- Angiomiolipoma (renal) & - Angiomiolipomas (renal) \\
- Quilotórax o ascitis quilosa & - Quilotórax \\
- Linfangioleiomioma & - Ascitis quilosa \\
- Adenomegalias por LAM & & \\
- ET probable o definitiva. & & \\
\hline
\end{tabular}

(Adaptado de la referencia 9).

LAM que infiltran linfáticos y quistes, con inmunohistoquímica positiva para alfa-actina, vimentina, desmina y HMB 45 (1-3).

\section{Manifestaciones radiológicas en el tórax}

\section{Parénquima pulmonar}

\section{Quistes}

Documentados en el $100 \%$ de los pacientes con LAM (14). Clásicamente son múltiples, simétricos y de morfología redonda u ovalada. En enfermedad severa pueden presentar morfologías poligonales (6). Se distribuyen de manera difusa en los pulmones y están rodeados por parénquima pulmonar normal $(8,15)$, En general presentan diámetros entre $2 \mathrm{y}$ $5 \mathrm{~mm}$, pero se han reportado quistes entre $0,5 \mathrm{~mm}$ y $10 \mathrm{~cm}$ (16). En estadios avanzados de la enfermedad el pulmón puede presentar cambios similares a los descritos en histiocitosis de células de Langerhans o enfisema centrilobulillar confluyente. El atrapamiento aéreo en la fase espiratoria en la tomografia computada (TC) no es común en pacientes con LAM, excepto en fases avanzadas, donde todo el parénquima pulmonar es reemplazado por quistes, El tamaño de los quistes normalmente disminuye en las imágenes en espiración, lo que confirma su relación con la vía aérea pequeña (14). Los quistes son más extensos en LAM-E que en LAM-CET (9). En LAM-CET las mujeres son más afectadas por quistes pulmonares que los hombres (17). La LAM se ha clasificado de acuerdo con la distribución y profusión de los quistes en: a) Grado 1 (leve). Área de quistes que compromete menos de un tercio del campo pulmonar.

b) Grado 2 (moderada). Entre un tercio y dos tercios del campo pulmonar.

c) Grado 3 (severa). Alteración mayor de dos tercios del campo pulmonar.

Las grados 2 y grado 3 sugieren el diagnóstico de LAM (15).

El diagnóstico diferencial de las enfermedades pulmonares quísticas en los estudios de imágenes incluye: histiocitosis de células de Langerhans, bronquiolitis folicular, neumonía intersticial linfocítica, neumonitis por hipersensibilidad, síndrome de Sjögren, síndrome de Birt-Hogg-Dubé, amiloidosis, metástasis pulmonares y enfisema $(1,3,15)$.

\section{Opacidades reticulares}

La radiografía de tórax puede ser normal o presentar opacidades reticulares finas, secundarias a interfases de la pared de los quistes con el parénquima pulmonar normal $(6,18)$. En algunos pacientes la TCAR puede evidenciar engrosamiento de septos interlobulillares y del intersticio peribroncovascular relacionados con edema intersticial por obstrucción de los vasos linfáticos (8).

\section{Aumento de la atenuación}

Los patrones de vidrio esmerilado y consolidación se presentan en el 8 al 14\% de los pacientes con LAM; se asocian con zonas de hemorragia alveolar 
y/o edema. Ocasionalmente, la atenuación en vidrio esmerilado puede corresponder a proliferación de músculo liso en las paredes alveolares $(6,8,14)$.

\section{Nódulos}

Están relacionados con hiperplasia de neumocitos tipo 2. Varían en tamaño $(1-10 \mathrm{~mm})$ y predominan en los lóbulos superiores (8-10).

\section{Manifestaciones pleurales}

\section{Neumotórax}

Es la forma de presentación en el 40 al 50\% de los pacientes con LAM. El neumotórax se presenta por la ruptura de quistes y puede ser recurrente. Se estima que 60 a- $80 \%$ de los pacientes con LAM tienen un episodio de neumotórax en el curso de su enfermedad (6). La pleurodesis puede prevenir su recurrencia, pero genera adherencias que dificultan un posible trasplante pulmonar. Las imágenes posteriores a la pleurodesis pueden mostrar calcificaciones y masas pleurales $(23$ y $14 \%$ de los pacientes respectivamente) (19).

\section{Quilotórax y quilopericardio}

El derrame pleural puede ser uni o bilateral, típicamente recurrente y aparece en el curso de la enfermedad $(9,14)$. En la radiografía de tórax el quilopericardio cursa con aumento de tamaño de la silueta cardiaca y en la TC con líquido pericárdico. En quilotórax los hallazgos son similares a los de la efusión pleural de otra naturaleza. En algunos pacientes, los coeficientes de atenuación del líquido pleural entre -50 y -100 UH, sugieren quilotórax. Sin embargo, en la mayoría de estos el quilotórax presenta densidades de líquido (14).

\section{Manifestaciones mediastinales}

\section{Adenomegalias mediastinales, dilatación del con- ducto torácico y linfagioleiomiomas mediastinales}

Se pueden encontrar adenomegalias en las diferentes estaciones mediastinales, con diámetro mayor de $1 \mathrm{~cm}$ en su eje corto. En el estudio reportado por Trotman et al., las adenomegalias hiliares fueron más comunes en los pacientes con LMA-CET (8).

En pocos casos puede evidenciarse dilatación del conducto torácico (diámetro normal menor de 2 $\mathrm{mm}$ ). El conocimiento del curso anatómico de esta estructura y los coeficientes de atenuación negativos pueden sugerir esta alteración (20).

Los linfangioleiomiomas son masas quísticas complejas con densidades entre 0 y $-25 \mathrm{UH}$ en TC (21).

\section{Manifestaciones radiológicas abdominales de la linfangioleiomiomatosis}

Las alteraciones abdominales en LAM (tanto en pacientes con LAM-CET y LAM-E) son frecuentes e incluyen: angiomiolipomas renales (54\%), adenomegalias (39\%) y linfangioleiomiomas (16\%). Con menor frecuencia se describen ascitis $(10 \%)$ y angiomiolipomas hepáticos (menos del 4\%) (21).

\section{Angiomiolipomas (AML) renales}

Corresponden a masas benignas de tamaño variable compuestas por grasa madura, vasos sanguíneos y músculo. Se presentan con mayor frecuencia en pacientes con LAM-CET (80-88\%), en comparación con pacientes con LAM-E (29-54\%). Son bilaterales en el $42 \%$ de los casos, y el sangrado es su complicación más importante $(19,21,22)$. El diagnóstico diferencial de AML renal debe realizarse con carcinoma renal (23). La ausencia de capa elástica en el componente vascular favorece el sangrado, especialmente en lesiones mayores de $4 \mathrm{~cm}$ o en aquellas con dilataciones aneurismáticas mayores de $5 \mathrm{~mm}$. En general, estos pacientes deben ser seguidos con ultrasonido anual cuando la lesión es menor de $4 \mathrm{~cm}$ y semestral en lesiones mayores (9).

\section{Angiomiolipomas hepáticos}

Estos tumores grasos benignos han sido reportados hasta en el 33\% de los pacientes con LAMCET. Los AML hepáticos son menos frecuentes en la variedad esporádica de la entidad (2\%). Los AML hepáticos de más de $4 \mathrm{~cm}$ tienen riesgo de sangrado y por tanto se debe realizar seguimiento imagenológico periódico (9). 


\section{Alteraciones linfáticas}

La infiltración ganglionar por las células LAM condiciona el aumento de tamaño ganglionar (adenomegalias) en el 39\% de los pacientes (21) y obstrucción linfática que origina masas quísticas complejas conocidas como linfangioleiomiomas (20-29\% de los casos) $(14,21)$. Como característica relevante los linfangioleiomiomas pueden presentar aumento de tamaño en el día (explicado por el incremento en el flujo linfático secundario a la actividad física). En el diagnóstico diferencial de estas lesiones deben considerarse enfermedades linfoproliferativas (19).

Los linfangioleiomiomas han sido descritos en pelvis, retroperitoneo, mediastino y linfáticos axiales y ocurren con mayor frecuencia en la variedad espontánea de LAM (29 vs. 9\%) (9, 24). La principal complicación de los linfangioleiomiomas es la ruptura, con salida de material quiloso a la cavidad peritoneal.

\section{Tamizaje}

En la población de alto riesgo para LAM (mujeres con CET, mujeres con neumotórax espontáneo, angiomiolipomas renales y/o enfermedad pulmonar quística difusa) deben realizarse estudios de tamizaje (15). Las guías ERS recomiendan tamizaje con TCAR de tórax en las mujeres con CET a los 18 años, que debe repetirse entre los 30 y 40 años, a menos que presenten síntomas respiratorios inexplicables (11). El tamizaje con TCAR en mujeres jóvenes no fumadoras con neumotórax espontáneo y en mujeres con angiomiolipomas renales o enfermedad pulmonar quística difusa, también es recomendable y costo-efectivo (15).

\section{Pronóstico y tratamiento}

La enfermedad no tiene tratamiento específico, por lo que evoluciona progresivamente hacia falla respiratoria. No obstante, el pronóstico de la LAM es variable. Se han descrito factores de mal pronóstico como presentación en mujeres premenopáusicas, debut con disnea, deterioro del intercambio gaseoso con un descenso importante en la DLCO, test de broncodilatador positivo, mayor proporción de parénquima afectado (medido en TCAR o histología), el de lesiones quísticas, presencia de hemosiderófagos en la biopsia pulmonar y neumotórax/quilotórax recurrentes (3). El embarazo y el uso de estrógenos $\mathrm{y}$ anticonceptivos orales puede empeorar la sintomatología de la enfermedad.

Para el manejo de la LAM se han utilizado diversas terapias basadas en los posibles mecanismos fisiopatogénicos de la enfermedad. La utilidad de las terapias con progesterona, agonistas liberadores de gonadotropinas (triptorelina y goserelina), doxiciclina, tamoxifeno e interferón alfa y la ooferectomía no ha sido concluyente en las diferentes series $(1,25)$. Estudios más recientes sugieren que la terapia con sirolimus y everolimus puede estabilizar la función pulmonar y reducir el volumen del quilotórax y los tamaños de los linfangioleiomiomas. Sin embargo, los pacientes presentan recaída al suspender el tratamiento $(5,26,27)$.

La opción terapéutica actual se basa en el trasplante pulmonar (1). Por otra parte, los pacientes con LAM requieren tratamiento de soporte con oxígeno, broncodilatadores, rehabilitación pulmonar, vacunación (influenza y pneumococo), manejo de las manifestaciones extrapulmonares (embolización de angiomiolipomas, dieta libre de grasas en quilotórax) y pleurodesis para neumotó$\operatorname{rax}(1)$. En la actualidad se encuentran en estudio inhibidores de la Rheb, antagonistas selectivos de estrógenos, inhibidores de la aromatasa, estatinas, inhibidores de la tirosinkinasa, inhibidores de las MMP, inhibidores de la angiogénesis e inhibidores de la linfangiogénesis $(3,28)$.

\section{Bibliografía}

1. Mavroudi M, Zarogoulidis P, Katsikogiannis N, Tsakiridis K, Huang H, Sakkas A, et al. Lymphangioleiomyomatosis: current and future. J Thorac Dis. 2013;5(1):74-79.

2. Cudzilo CJ, Szczesniak RD, Brody AS, et al. Lymphangioleiomyomatosis screening in women with tuberous sclerosis. Chest. 2013;144(2): 578-585.

3. Taveira-DaSilva AM, Moss J. Clinical features, epidemiology, and therapy of lymphangioleiomyomatosis. Clinical Epidemiology 2015;7 249-57.

4. Grzegorek I, Drozdz K, Podhorska-Okolow M, Szuba A, Dziegiel P. LAM cells biology and lymphangioleiomyomatosis. Folia histochemica et cytobiologica. 2013;51:1-10. 
5. Henske EP, McCormack FX. Lymphangioleiomyomatosis a wolf in sheep's clothing. J Clin Invest. 2012;122(11):380716.

6. Jawad H, Walker CM, Wu CC, Chung JH. Cystic interstitial lung disease: recognizing the common and uncommon entities. Curr Probl Diagn Radiol. 2014:115-27.

7. Lee K-H, Lee JS, Lynch DA, Song KS, Lim TH. The radiologic differential diagnosis of diffuse lung disease characterizes by multiple cysts or cavities. J Comput Assist Tomogr. 2002;26(1):5-12.

8. Trotman-Dickenson B. Cystic lung disease: achieving a radiologic diagnosis. Eur J Radiol. 2014;83:39-46.

9. Avila NA, Dwyer AJ, Rabel A, Moss J. Sporadic lymphangioleiomyomatosis and tuberous sclerosis complex with lymphangioleiomyomatosis: comparison of CT features. Radiology. 2007;242(1):277-85.

10. Seaman DM, Meyer CA, Gilman MD, McCormack FX. Diffuse cystic lung disease at high resolution CT. AJR 2011;196:1305-11.

11. Johnson SR, Cordier JF, Lazor R, Costbel U, Harari S, Reynoud-Gaubert $\mathrm{M}$ et al. European Respiratory Society guidelines for the diagnosis and management of lymphangioleiomyomatosis. Eur Respir J. 2010;35(1):14-26.

12. Young LR, Vandyke R, Gulleman PM. Serum vascular endothelial growth factor-D prospectively distinguishes lymphangioleiomyomatosis from other diseases. Chest. 2010;138:674-81.

13. Young L, Lee HS, Inoue Y, Moss J, Singer LG, Strange C, et al. Serum VEGF-D a concentration as a biomarker of lymphangioleiomyomatosis severity and treatment response: a prospective analysis of the Multicenter International Lymphangioleiomyomatosis Efficacy of Sirolimus (MILES) trial. Lancet Respir Med. 2013;1(6):445-52.

14. Pallisa E, Sanz P, Roman A, Majó J, Andreu J, Caceres J. Lymphangioleiomyomatosis: pulmonary and abdominal findings with pathologic correlation. RadioGraphics. 2002;22:S185-98.

15. $\mathrm{Xu} \mathrm{KF}$, Lo BH. Lymphangioleiomyomatosis: differential diagnosis and optimal management. Ther Clin Risk Management. 2014;10:691-700.
16. Barrera EA, Mancheño N, Vera-Sempere F, Padilla J. Lymphangioleiomyomatosis. Arch Bronconeumol. 2011;47(2):85-93.

17. Adriaensen MEPM, Schaefer-Prokop CM, Duyndam DAC, Zonnenberg BA, Prokop M. Radiological evidence of lymphangioleiomyomatosis in female and male patients with tuberous sclerosis complex. Clinical Radiology. 2011;66:625-8.

18. Hohman DW. Noghrehkar D, Ratnayak S. Lymphangioleiomyomatosis: a review. Eur J Int Med. 2008(19):319-24.

19. Avila NA, Dwyer AJ, Moss J. Imaging features of lymphangioleiomyomatosis: diagnostic pitfalls. AJR. 2011;196:982-6.

20. Liu ME, Branstetter BF, Whetstone J, Escott EJ. Normal appearance of the distal thoracic duct. AJR. 2006;197:1615-20.

21. Avila, NA, Kelly JA, Chu SC, Dwyer AJ, Moss J. Lymphangioleiomyomatosis: abdominopelvic CT and US findings. Radiology. 2000;216:147-53.

22. Ryu JH, Moss J, Beck GJ, Lee JC, Brown KK, Chapman JT et al. The NHLBI lymphangioleiomyomatosis registry. characteristics of 230 patients at enrollment. Am J Respir Crit Care Med. 2006;173:105-11.

23. Hindman N, Ngo L, Genega EM, Melamed J, Wei J. Angiomyolipoma with minimal fat: can it be differentiated from clear cell renal cell carcinoma by using standard MR techniques? Radiology. 2012;265(2):468-77.

24. Harari S, Torre O, Moss J. Lymphangioleiomyomatosis: what do we know and what are we looking for? Eur Respir Rev. 2011;20(119):34-44.

25. Casanova A, Ancochea J. Lymphangioleiomyomatosis: new therapeutic approaches. Arch Bronconeumol. 2011;47:57980 .

26. McCormack FX, Inoue Y, Moss J. National Institutes of Health Rare Lung Diseases Consortium; MILES Trial Group. Efficacy and safety of sirolimus in lymphangioleiomyomatosis. N Engl J Med. 2011;364(17):1595-606.

27. Bissler JJ, Kingswood JC, Radzikowska E. Everolimus for angiomyolipoma associated with tuberous sclerosis complex or sporadic lymphangi- oleiomyomatosis (EXIST-2): a multicentre, randomised, double-blind, placebo-controlled trial. Lancet. 2013;381(9869):817-24.

28. Taveira-DaSilva AM, Moss J. Management of lymphangioleiomyomatosis. F1000Prime Reports. 2014;6(116):1-16. 